\title{
NOTE ON THE EFFECT OF REPEATED STRESSES ON THE MAGNETIC PROPERTIES OF STEEL
}

\author{
By M. F. Fischer
}

\begin{abstract}
Changes in the magnetic properties of steel generally accompany changes in the other physical characteristics. This suggests the possibility of detecting and following the progress of a fatigue crack by magnetic methods. It was found, however, that the magnetic changes produced by repeated stresses above the endurance limit are not of a type which can be associated with the characteristic effect of a crack transverse to the direction of magnetization. The similarity of the observed magnetic changes with those brought about by the removal of externally applied static stresses indicates that the observed changes may be produced by the partial relief or redistribution of initially existing internal stresses. The fact that the magnetic changes brought about by understressing are of a similar character though less in magnitude suggests that some of the beneficial effect of understressing may be due to partial stress relief.
\end{abstract}

\section{CONTENTS}

I. Introduction

II. Material and apparatus _._. 722

1. Material _... 722

2. Testing machine and specimens_._. 722

3. Magnetic testing apparatus_._.

4. Methods of measurement.

III. Observations and results__._.

IV. Discussion

V. Summary and conclusions_.......... 732

\section{INTRODUCTION}

Burrows ${ }^{1}$ once made the statement that a magnetic method may be expected to follow the progress of a fatigue failure in iron or steel. While magnetic analysis has in some cases been successfully used in the detection and study of changes in the physical characteristics of such materials, there is no evidence to substantiate the prediction that it may be successfully applied to the detection of fatigue cracks. Except for the result of a single experiment by Sanford, ${ }^{2}$ no evidence is available to indicate that repeated stresses have any permanent

1 C. W. Burrows, "Some applications of magnetic analysis to the study of steel products," Proc. A. S. T. M., 17, pt. $2 ; 1917$.

${ }^{2}$ R. L. Sanford, Nondestructive Testing of Wire Hoisting Rope by Magnetic Analysis, B. S. Tech, Paper No. $315 ; 1926$. 
effect on the magnetic properties of steel. The present work was undertaken, therefore, for the purpose of studying the character of the changes in magnetic properties resulting from the application of repeated stresses and to determine whether or not such changes bear a sufficiently close relationship to the progress of a fatigue failure to warrant further investigation of the possibilities of a magnetic method for the study of fatigue phenomena. ${ }^{3}$

\section{MATERIAL AND APPARATUS}

\section{MATERIAL}

Two readily available types of commercial steel were used for a preliminary survey. The samples were taken from two lots of drill rod having about 1 per cent carbon and one lot of cold-rolled machinery steel having a carbon content of approximately 0.13 per cent. The two lots of drill rod showed somewhat different magnetic characteristics and were designated as lot 1 and lot 2 .

Had the results of the preliminary work held out hope of following the progress of a fatigue failure by magnetic methods, it would have been necessary to extend the work to a wider range of materials to determine, for purposes of comparison, the endurance limits, static tensile properties, complete chemical composition, metallographic structure, etc., and to study the effect of annealing. Since the magnetic method, as will be shown hereafter, did not follow the progress of the fatigue failure on one hard and one soft steel, it was unnecessary to go to the wider range of materials or to define more closely the materials that were used.

\section{TESTING MACHINE AND SPECIMENS}

Figure 1 is a photograph of the R. R. Moore ${ }^{4}$ rotating-beam testing machine used. Transverse loading causes the longitudinal fibers of the test specimen to be alternately in compression and tension as the specimen rotates. The machine runs at 2,000 r. p. m. and is provided with a revolution counter and an automatic cut-off switch which stops the machine on failure of the specimen.

The specimens used in this machine are easily adapted to magnetic tests. While certain detailed dimensions and form are necessarily fixed by the method of clamping in the testing machine, the general form of the test section of the specimen can be made to suit the specific test requirements. Specimen A of Figure 2 is of the type generally used in the study of endurance properties. The stresses

\footnotetext{
${ }^{3}$ The recent book by H. F. Moore and J. B. Kommers, The Fatigue of Metals, McGraw-Hill; 1927, describes in detail the many theories of fatigue failure and also contains an extensive bibliography on the subject of fatigue.

4 R. R. Moore, “Some fatigue tests on nonferrous metals," Proc. A. S. T, M., 25, 1925.
} 
B. S. Journal of Research, RP26

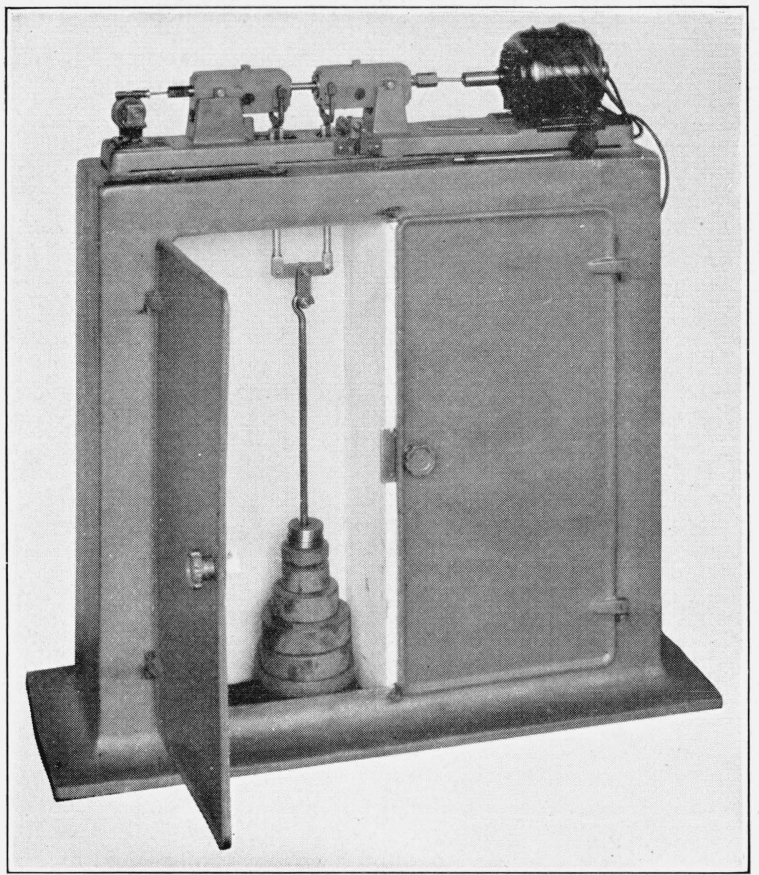

FIG. 1.-Rotating beam testing machine

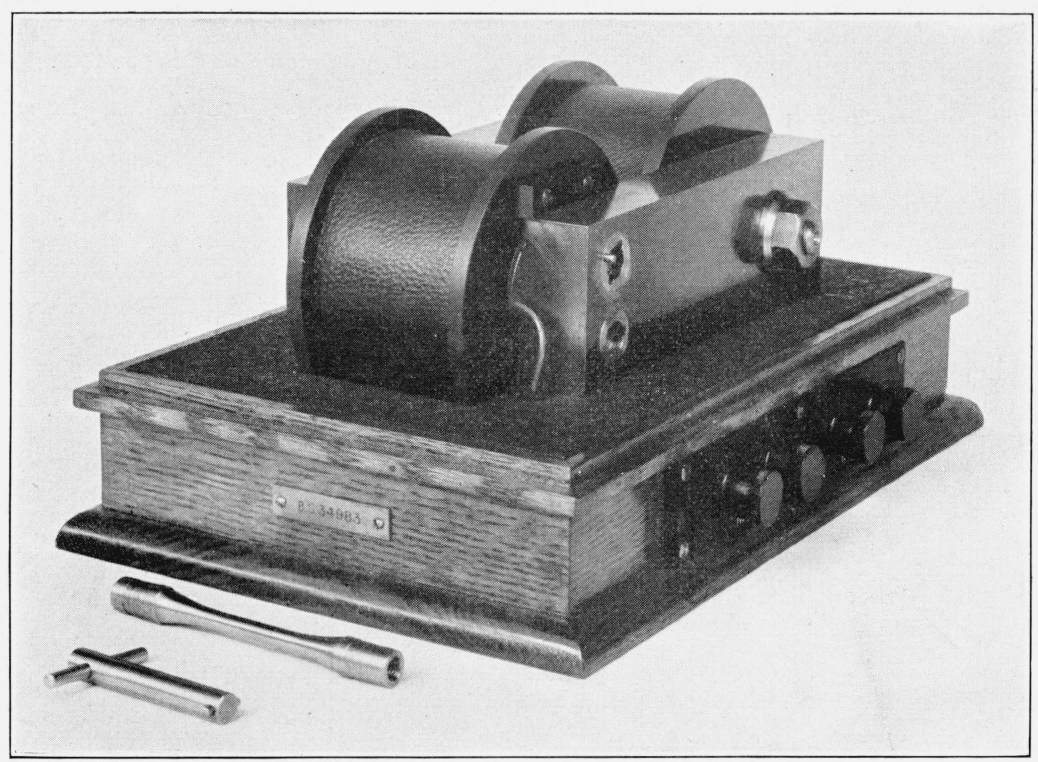

FIG. 3.-Magnetic comparator 
are greatest at the minimum section, and consequently only a very small part of the material is subjected to the maximum stress range. In order to have a greater proportion of the sample subjected to the maximum stress range, a specimen was designed having a uniform section about 1 inch long. This type of specimen ( $B$ in fig. 2) was used for the greater portion of the tests. All test specimens were polished longitudinally to remove circumferential scratches, and none broke at the fillet.

The sample designated as $C$ in Figure 2 was designed to permit of making magnetic tests without
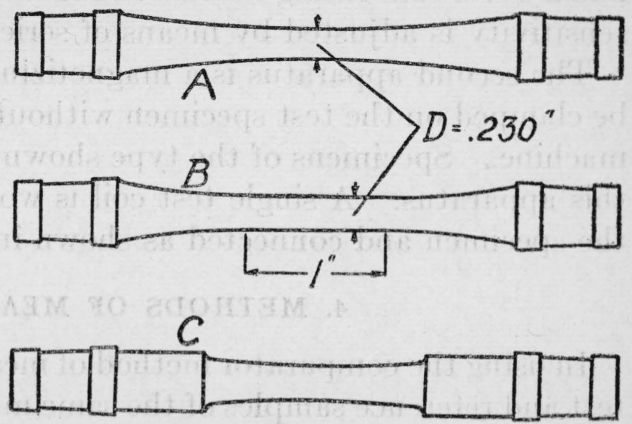

FIG. 2.-Test specimens

removal from the machine. The cylindrical portions near the ends fit into a removable magnetizing yoke.

\section{MAGNETIC TESTING APPARATUS}

Magnetic measurements can be made by any of several methods. Of the two methods used in the present work, the one by means of which most of the following data were obtained is a modification of the magnetic comparator previously developed and described by the writer ${ }^{5}$ for making magnetic measurements on short samples. In

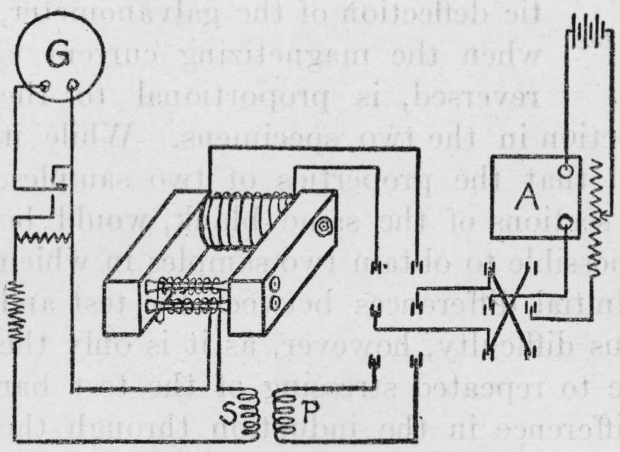

FIG. 4.-Electrical connections of the comparator circuit this apparatus the sample under examination is compared with a second, sample of similar material and properties which is designated as the reference bar. As shown in Figure 3 , the comparator consists essentially of an electromagnet with poles and yoke of soft iron. Symmetrically located near the ends of each pole are two holes into which fit the bushings throughwhich contact between the test samples and poles is made. Magnetizing solenoids surround both the yoke and test samples, and test coils of 1,000 turns each surround the test and reference bars. The electrical connections are shown in Figure 4, from which, for the sake of clear-

\footnotetext{
${ }^{5}$ M. F. Fischer, Apparatus for the Determination of the Magnetic Properties of Short Bars, B. S. Sci.
} Paper No. 458, 1922. 
ness, the magnetizing solenoid over the specimens is omitted. The two magnetizing solenoids are connected in series, aiding, through a reversing switch, ammeter, and control rheostats to a storage battery. The two test coils are connected in series, opposing, through the secondary of a calibrating mutual inductance to the galvanometer whose sensitivity is adjusted by means of series and parallel resistances.

The second apparatus is a magnetizing yoke of soft iron which can be clamped on the test specimen without removing it from the testing machine. Specimens of the type shown in Figure $2 C$ were used with this apparatus. A single test coil is wound on the central section of the specimen and connected as shown in Figure 5.

\section{METHODS OF MEASUREMENT}

In using the comparator method of measuring the magnetic changes, test and reference samples of the same material are placed in the upper

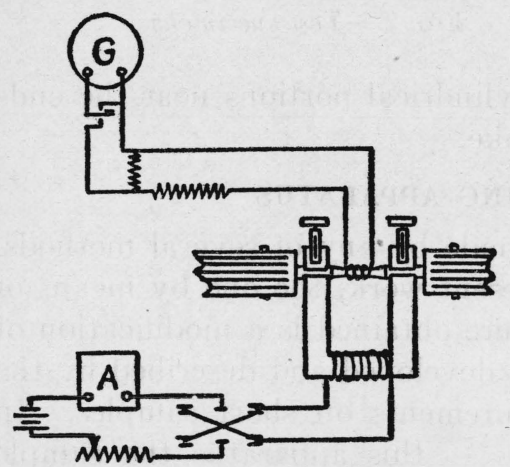

FIG. 5.-Electrical connections of the single-yoke circuit and lower holes, respectively, in the ends of the pole pieces. The reference sample is left in position during the test of all samples of similar material. It can be assumed, without appreciable error, that the same magnetizing force acts simultaneously on both samples when current is flowing in the magnetizing coils. With the test coils properly connected, the ballistic deflection of the galvanometer, when the magnetizing current is reversed, is proportional to the difference in magnetic induction in the two specimens. While it might ordinarily be expected that the properties of two samples, cut from possibly adjoining sections of the same blank, would be the same, it is practically impossible to obtain two samples in which such identity exists. Small initial differences between the test and reference bars cause no serious difficulty, however, as it is only the change in this difference due to repeated stressing of the test bar which is important. The difference in the induction through the bars at corresponding magnetizing forces is measured directly by the comparator. Changes in induction due to any treatment are designated as $\Delta B$. Observations of similar differences in induction for a series of magnetizing currents were made on each test sample before subjecting it to repeated stresses. Measurements were made at the same values of magnetizing currents after each period of stressing. Errors due to possible differences in contact reluctance between the test sample and magnetizing yoke were minimized by 
taking the average of several sets of readings, between which the sample was removed from the comparator and reinserted.

In the second method of measurement, using the single yoke, readings were made of the total induction corresponding to a series of magnetizing currents. These tests were made with the load removed. Although the precision of the measurement of the changes was lower in this case than with the comparator, similar results were obtained by both methods. The fact that it is unnecessary to remove the sample from the testing machine for the magnetic measurements is a decided advantage, and the precision of the single-yoke method can probably be improved by a suitable modification of the apparatus.

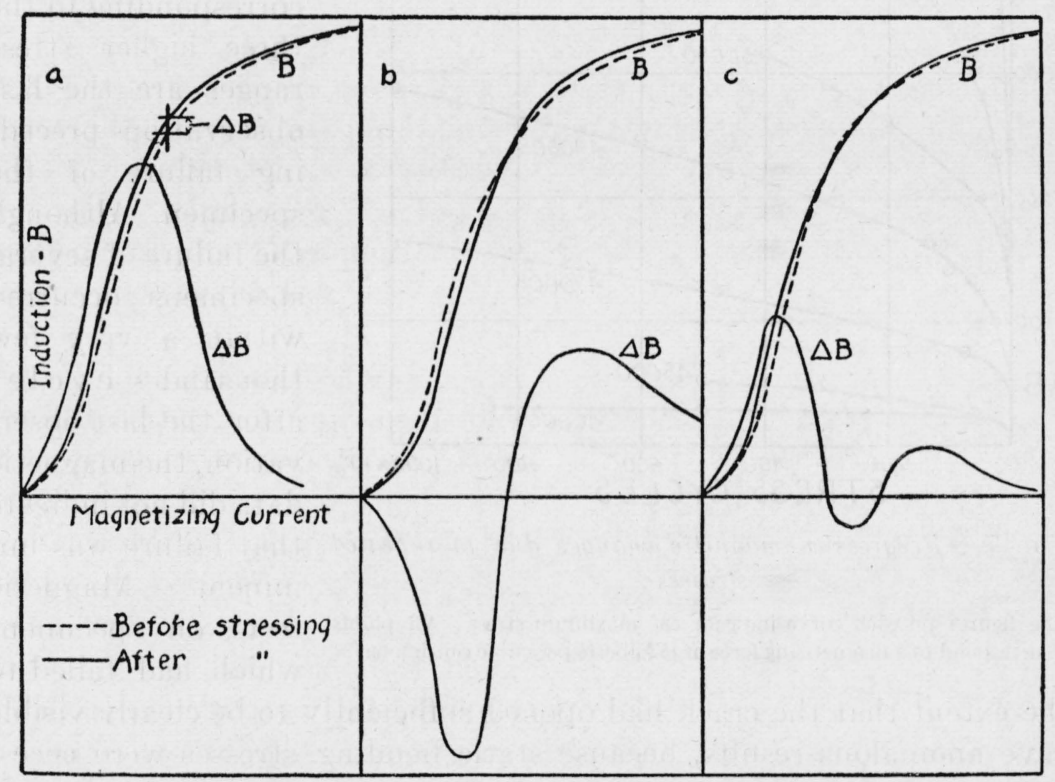

FIG. 6.-Characteristic changes in the magnetic induction effected by repeatedly stressing samples of different materials

\section{OBSERVATIONS AND RESULTS}

The characteristic magnetic changes brought about by the cyclic application of stress are graphically represented by the curves of Figure 6. The dashed lines are the normal induction curves of the unstressed samples. The full lines are the normal induction curves of the same samples after the application of repeated stresses. The changes in induction are represented by the $\Delta B$ curves, which are drawn to a larger scale. Curve $(a)$ is representative of the drill rod of lot 1 , curve $(c)$ of the drill rod of lot 2 , and curve $(b)$ of the coldrolled steel. For any single value of magnetizing force and type of material, the magnitude of the changes depends both on the value of applied stress and the number of applications. 
The samples of drill rod from lot 1 gave, without exception, results of the character indicated by the curves of Figure $6(a)$. The effects of the magnitude of the stress and the number of its applications are

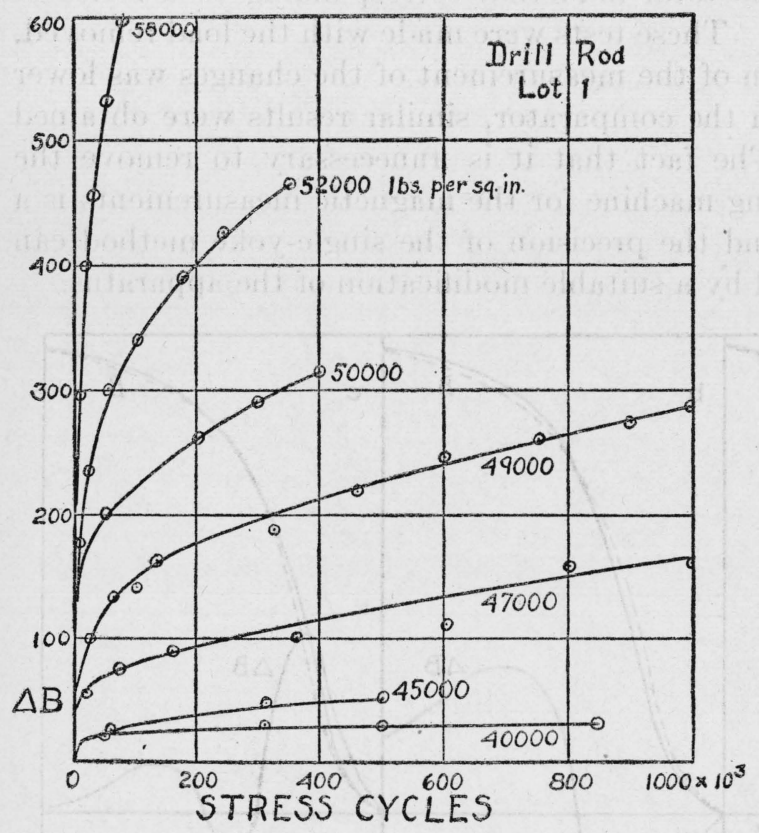

FIG. 7.-Progressive magnetic changes due to repeated stresses

The figures on each curve indicate the maximum stress. All points correspond to a magnetizing force of 15 gilberts per cubic centimeter gave anomalous results, because static bending stresses were neces-

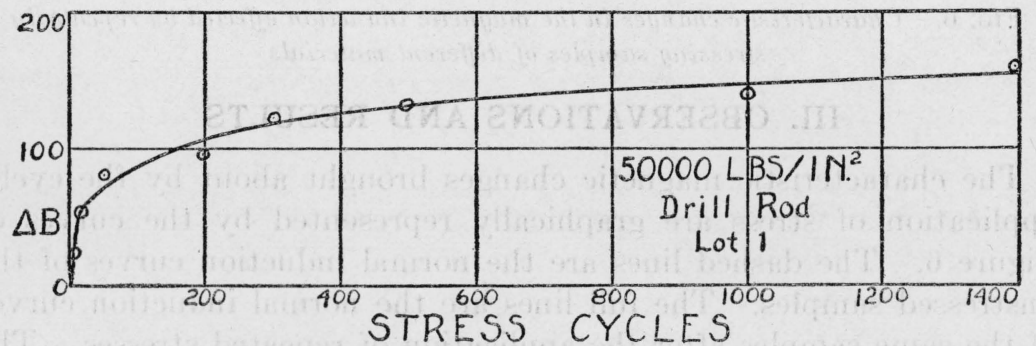

FIG. 8.-Magnetic changes occurring during the initial cycles of repeated stressing

sarily applied in placing the specimen in the comparator, thus superposing on the normal effects those of the applied static stresses.

Figure 8 shows the magnetic changes in a specimen which was initially rotated by hand and the first observation made after five 
cycles of stress. It is probable that some magnetic change would have been observed even after a single cycle.

Figure 9 is taken from the data of Figure 7 and shows the magnetic changes as a function of the maximum stress range. The

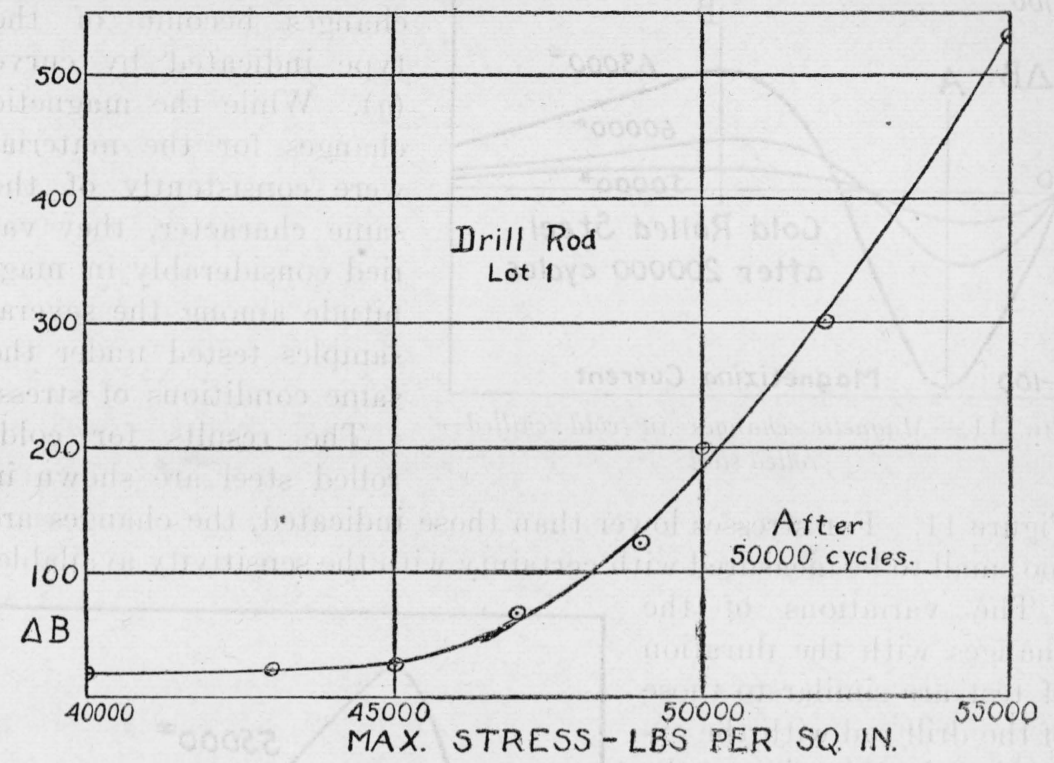

FIG. 9.-Magnetic changes as a function of the maximum stress

magnetic changes are those observed after the application of 50,000 cycles of stress to each of the several specimens.

It is known that understressing, in most cases, raises the endurance limit. A sample of this material was run for $10,000,000$ cycles at

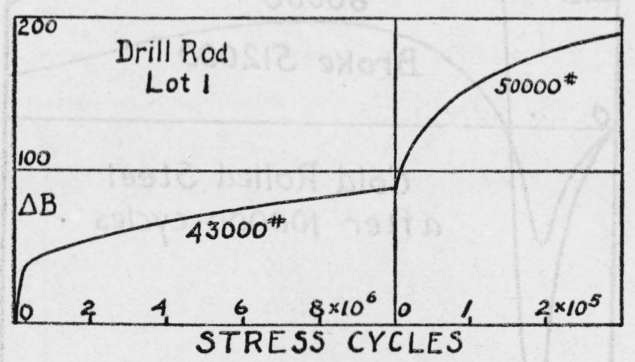

FIG. 10.-Effect of two different stresses applied successively to the same sample

Magnetizing force 15 gilberts per cubic centimeter $43,000 \mathrm{lbs}$./in. ${ }^{2}$ without evidence of failing and then run at a stress of 50,000 lbs./in. ${ }^{2}$ The resulting magnetic changes are shown in Figure 10. The magnetic changes occurring after the application of the higher stress are smaller than those observed in other samples which had been tested at the higher stress alone, although the total change in the present case is of the same order as would have been produced by the higher stress. It is probable that the effect of successively increasing stresses is cumulative, the magnetic changes produced by each depending on the magnitude and number of cycles of application of all preceding stresses. 
Magnetic changes produced in drill rod of lot 2 are similar to those indicated in curves $(a)$ and $(c)$ of Figure 6 . The changes taking place in the early part of the test are of the type shown in curve $(c)$.

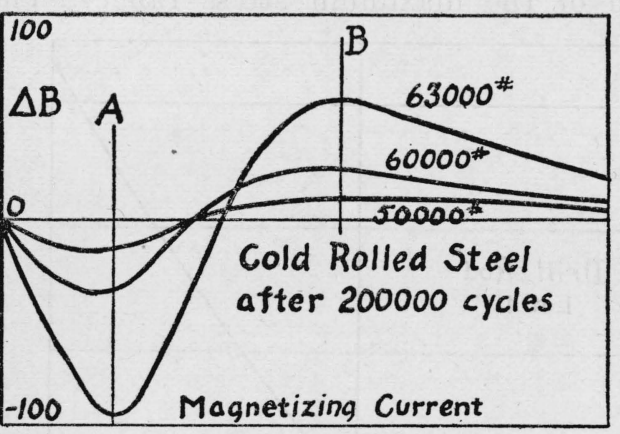

Fig. 11.-Magnetic changes in cold chilledrolled steel

As the test proceeds the changes become of the type indicated by curve (a). While the magnetic changes for the material were consistently of the same character, they varied considerably in magnitude among the several samples tested under the same conditions of stress.

The results for coldrolled steel are shown in

Figure 11. For stresses lower than those indicated, the changes are too small to be measured with certainty with the sensitivity available.

The variations of the changes with the duration of test are similar to those of the drill rod with the exception that the sign of the changes is negative for magnetizing forces in the region $\mathrm{A}$ and positive for magnetizing forces in the region $\mathrm{B}$. In Figure 12 the magnetic changes in the sample stressed at 55,000 pounds are much larger than those in the sample stressed at 60,000 pounds, which is contrary to what had heretofore been observed under the same comparative conditions of test. As is evidenced by the relative number of cycles to failure of the two samples, considerable difference may have existed in the initial conditions of the

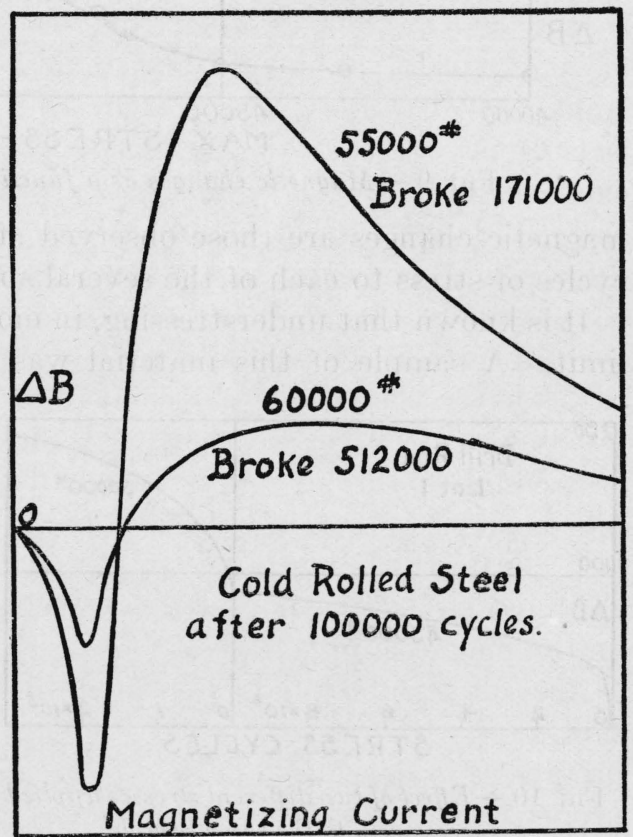

FIG. 12.-Effect of the initial condition of the material on the magnetic changes and endurance properties two samples. Similar variation in the endurance properties of apparently identical materials is frequently observed in fatigue testing. 
The fact that at stresses below $50,000 \mathrm{lbs} . / \mathrm{in} .^{2}$ repeated stress showed no effect upon the magnetic properties of the cold-rolled steel is sufficient to negative the suggestion that arises on looking at Figures 7 and 10, for drill rod, which, by themselves, suggest that the study of magnetic changes might offer an accelerated method of approximately determining the endurance limit.

The endurance limit of the drill rod, lot 1 (as indicated by the breaking of specimens loaded at $50,000 \mathrm{lbs}$./in. ${ }^{2}$ or above, at a few hundred thousand cycles, while a specimen loaded at 43,000 lbs./in. ${ }^{2}$ apparently has an indefinite life, fig. 10), can not be far from 45,000 lbs./in. ${ }^{2}$ The agreement of this figure with the change of slope in Figure 9 might justify hope for an accelerated testing method, but since a similar curve for the cold-rolled steel would show the change of slope only at 50,000 lbs./in. ${ }^{2}$ or higher, and the endurance limit of 0.13 per cent $\mathrm{C}$ cold-rolled steel is obviously not so high as that, the hope is not realized.

\section{DISCUSSION}

It is evident from the preceding data that the magnetic properties of some steels are modified by repeated stresses of the type here employed. While the changes are small in most cases, the evidence is conclusive that they are real and permanent. In not a single case, however, are the changes of a character which can be directly associated with the magnetic effects of a crack transverse to the direction of magnetization. Since such a crack constitutes a discontinuity in the magnetic circuit, it would naturally be expected that its presence would cause the apparent magnetic permeability to be lower than that of the unaffected material for all values of induction. In all cases, however, the observed permeability is increased either throughout the whole range of inductions or in certain parts of the induction curve. It is quite probable, therefore, that any effect which might be produced by the presence of cracks is entirely masked by another effect which has a much greater influence on the magnetic properties.

According to prevailing ideas, a fatigue failure starts at a relatively slow rate with respect to the number of repetitions of stress and progresses at a continually increasing rate. The change in magnetic properties starts at the maximum rate and progresses at a continually decreasing rate. This is added evidence that the magnetic change does not bear a direct relation to the progress of the fatigue failure.

It has long been known that the magnetic properties of iron and steel are modified by the application of a static load to the test material. The character and magnitude of such changes depend on the type and magnitude of the stresses, and also on the degree to 
which the test sample is magnetized. Figure 13 shows the characteristic magnetic changes which occur in ferrous materials when subjected to static stresses. The ordinates are the changes in magnetic induction due to the load and the abscissas are the magnetizing currents at which the changes are measured.

Curve $(a)$ indicates the characteristic magnetic changes due to either a high tensile stress or to a compressive stress of any magnitude. The magnetic changes under these conditions are negative for all magnetizing forces.

Low tensile stresses produce changes of the style represented by curve (b). It is to be observed in this curve that the magnetic changes are positive for low values of magnetizing force but become negative when the magnetizing. force is increased. When the

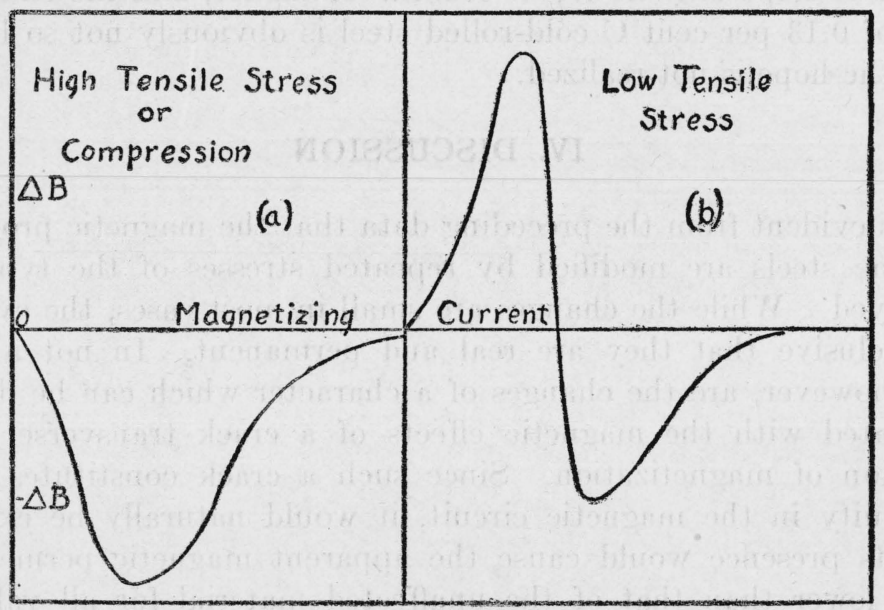

FIG. 13.-Changes in the magnetic induction $(\Delta B)$ due to the application of static stresses

magnetizing forces are increased indefinitely, the magnetic changes due to stresses of any type become vanishingly small.

If the applied stresses exceed the elastic limit of the material, permanent magnetic changes of the type shown in Figure 13 (a) occur.

Comparison of the curves of Figure 13 and Figure 6 ( $a$ and $b$ ) shows that the magnetic changes due to static stress and those brought about by repeated stresses differ mainly in sign. If the data on static stresses had been plotted with signs reversed, thus indicating the effect of stress relieval, it would be seen that the magnetic changes due to repeated stresses are exactly similar in character to those corresponding to the removal of a previously existing static stress. From this it appears that the observed changes in magnetic properties which accompany the application of repeated 
stresses are probably associated with the relief or redistribution of initial stresses rather than with the start and development of the crack which ultimately results in failure.

The probability of a change in magnitude or redistribution of initial stresses is suggested by Gough and Hanson, ${ }^{6}$ and others in their respective theories of fatigue failure. Direct evidence of the relief of internal stresses by repeated understressing is furnished by some "turning-down" tests made by Moore and Jasper. ${ }^{7}$ Strain changes resulting from the removal of surface material were generally smaller in specimens which had previously been understressed than in virgin material.

As shown by the lowest curve of Figure 7, the magnetic changes produced by understressing differ only in magnitude from those effected by overstressing and, therefore, probably have their origin in changes similar to those produced by the higher stresses. The fact that partial stress relief may have occurred under these circumstances suggests that some of the improvement in endurance properties resulting from understressing may be due to such stress relief.

It is to be borne in mind that under cycles of flexural stresses the outside fibers of the test specimens are subjected to the greatest stresses, the maximum stresses in the concentric lamellæ of the test piece being proportional to the cubes of their respective radii and varying from the maximum, both in tension and compression, to zero during each revolution of the specimen. Since the stresses vary across the section in this manner, it is reasonable to suppose that any effect which they might have on the properties of the test bar also varies in an approximately similar manner and that the observed magnetic changes are the resultant of those occurring in all the lamellæ of the test bar. In Figure 9 it is seen that the magnetic changes corresponding to a maximum stress of 43,000 pounds are small. For a maximum stress of 50,000 pounds, the magnetic changes are moderately large. In the latter case, however, only about 10 per cent of the cross-sectional material is stressed above 43,000 pounds, and it is probable, therefore, that the difference in the magnetic changes corresponding to these maximum stresses is contributed almost entirely by the small area in which the stresses exceeded the lower value.

Differences in the character of the magnetic changes which occurred. in the several materials studied may be attributed to differences in the magnitude or distribution of the initial internal stresses. The magnetic changes were mainly of the two types shown in curves $(a)$ and $(b)$ of Figure 6 . Curve $(c)$ of this figure may be considered as a

\footnotetext{
${ }^{6}$ H. J. Gough and D. Hanson, “The behavior of metals subjected to repeated stresses," Proc. Roy. Soc., 104A; 1923.

${ }^{7}$ H. F. Moore and T. M. Jasper, “An Investigation of the Fatigue of Metals.” Bull. No, 142, Eng. Exp. Sta. Univ, of Ill.; 1923,
} 
combination of curves $(a)$ and $(b)$. It is not to be supposed that a change of a single type occurs without a compensatory change of another type, but rather that one change, by reason of its magnitude or the volume of material involved, has a predominating influence on the magnetic properties.

\section{SUMMARY AND CONCLUSIONS}

The results of the present study may be summarized as follows:

1. Repeated stresses of the reversed-bending type affect the magnetic properties of the materials studied.

2. The magnetic changes accompanying repeated stresses are not characteristic of the effect of a fatigue crack transverse to the direction of magnetization.

3. Imminence of failure is not indicated by the magnetic changes.

4. The character of the magnetic changes differs according to the material of which the test sample is made.

5. The magnitude of the magnetic changes for a specific material is a function of the applied stress, the number of cycles of its application, and the values of the magnetizing force at which the changes are observed.

Since the magnetic changes which have been observed can not be associated with a fatigue crack, a basis of explanation was sought in the known effects of static stresses on the magnetic properties of ferromagnetic materials. From the similarity of the observed magnetic changes resulting from the removal of static loads from the test material, the following tentative conclusions appear to be warranted:

1. The magnetic changes brought about by repeated stresses are such as would be caused by the partial relief or redistribution of initially existing internal stresses.

2. The magnetic effects of such stress relief are so large as to mask the small magnetic changes which may directly result from a fatigue crack.

3. The fact that magnetic changes are produced by repeated stresses even below the endurance limit suggests the probability that understressing results in the partial relief or redistribution of the internal stresses. Some of the beneficial effect of understressing may be explained on this basis.

4. Differences in the character of the magnetic changes observed in the different materials can be associated with the difference in type, magnitude, or distribution of the partially relieved stresses.

Washington, May 16, 1928. 